\title{
New method of digital modulative adaptative auto-calibration of infrared imaging devices
}

\author{
by V.M. SAPTZIN (*), V.I. KOBER $\left({ }^{* *}\right)$ and V.P. VAVILOV $(* * *)$
}

(") Heat and Mass Transfer Inst. P. Brovki, 15, 220728, Minsk, Belarus.

("*) Inform. Transmission problems Inst. Ermolovoy, 19, Moscow, Russia.

("* *) Tomsk Polytechnic University, Lenina, 30, Tomsk, Russia.

\begin{abstract}
This paper describes the new method of digital auto-calibration IR imaging systems based on using low-amplitude 2D cyclic scanning and solving multi-dimensional inverse problem. The method does not require the use of reference bodies and stages of registration and calibration can coincide. Mathematical algorithms require $\approx 100 \mathrm{~N}^{2}$ operations and are realized by using IBM PC/AT for $N<256$.
\end{abstract}

\section{Introduction}

Modern technology allows to develop IR imaging systems with noise equivalent temperature difference (NETD) of about $0.001 \mathrm{~K}[1]$ but the practical realization of these systems and their metrology encounters some problems [2 to 4]. Nonuniformity of $I R$ registrating device is one of these problems to be discussed in the present paper.

Non-uniform sensitivity of IR camera can be due to non-identical elements in the arrays, optical distortions and background irradiation. Digital correction of these effects is possible if the relationships between IR radiation intensity $l_{i, j}$ and output electrical signals $a_{i, j}$ are known. In linear approximation:

$$
a_{i, j}=g_{i, j} \cdot l_{i, j}+h_{i, j} \quad 1 \leq i, j \leq N
$$

where $N$ is the number of pixels per line or column.

Coefficients $g_{i, j}$ and $h_{i, j}$ are usually determined by use of calibration procedure with two reference bodies, having intensities /1 and 12. Unfortunately, for systems with wide spectral and dynamic range and NETD $<0.1 \mathrm{~K}$, this procedure has some drawbacks.

The best IR arrays use the InSb and CdHgTe detectors [5 and 6] but the attempts to enhance their uniformity, stability and sensitivity up to theoretical limits have still been unsuccessful. Better results were obtained on the base of Simade Schottky diodes arrays [7]. In general, they provide worse than InSb detectors sensitivity but much better uniformity and lack of low-frequency noise [8 and 9]. But even in this case the random variations of spectral sensitivity from one detector to another will limit the temperature resolution of IR systems [10].

It is important that such a problem arises even for single - or few - detectors arrays, for example when high metrological accuracy is needed.

We suggest the new method of adaptive auto-calibration which uses the ideas of modulative spectroscopy [11] and methods of solution for non-correct multidimensional inverse problems [12]. The method does not require the use of reference bodies and the stages of registration and calibration can coincide. 


\section{Discussion of inverse problem}

The basic idea is to use the low-amplitude two-dimensional (2D) cyclic scanning of the IR camera itself or view angle scanning with additional mirror mounted on the camera. This idea is not new for image processing [13] but the basic problem is choosing of scanning law as simple as possible which permit from minimum number of images to determine correctly all $g_{i, j}, h_{i, j}$ and $l_{i, j}$ without any essential assumptions about its space characteristics.

The working analog of such a system with $N \approx 10^{4}$ is probably the human's eye. During observing the eye do many moves and fast low-amplitude $2 \mathrm{D}$ scanning with frequency $\approx 30-60 \mathrm{~Hz}$ (tremor) is one of them [14]. Researches in this field last more than fifty years [14 to 16] but the role of tremor in visual information processing is not clear still.

As the basis we have used the simplest 2D cyclic discrete scanning consisting of 3 points. Each pixel is moving for one pixel up or down and one pixel left or right. For this scanning consequence of 3 basic images:

$$
\left\{a_{i, j}\right\},\left\{b_{i, j}\right\},\left\{c_{i, j}\right\}
$$

minimum necessary for further processing can be obtained. Unfortunately, restoration of full input information $\left\{I_{i, j}\right\}$ is possible if at least one matrix of coefficients $\left\{g_{i, j}\right\},\left\{h_{i, j}\right\}$ is known or has small dispersion. In a case of essential dispersion of both matrices, it is necessary to make two measurements of an object under the following condition:

$$
11<12
$$

For absolute and independent calibration of IR system it is enough to place two small-size reference emitters with different $/ 1$ and $/ 2$ into the frame.

\section{Equations system and algorithms}

According to (1-3) and scanning law an initial system of equations for $4 N^{2}+4 N$ unknown quantities $g_{i, j}, h_{i, j}, 11_{i, j}$ and $12_{i, j}$ can be written as following:

$$
\begin{array}{ll}
g_{i, j} . K_{i, j}+h_{i, j}=a K_{i, j} & K=1,2 \\
g_{i, j} . K_{i+1, j}+h_{i, j}=b K_{i, j} & 1 \leq i, j \leq N \\
g_{i, j} . K_{i, j+1}+h_{i, j}=c K_{i, j} & g_{1,1}=G^{-1}, h_{1,1}=G^{-1} . H
\end{array}
$$

where constants $G>0$ and $H$ are determined by the scale of $l_{i, j}$.

For correction of non-uniformity it is enough to suppose $G=1, H=0$. It is important that the structure of system (4) permits to split procedures of restoration $\left\{g_{i, j}\right\},\left\{h_{i, j}\right\},\left\{/ 1_{i, j}\right\}$ and $\left\{/ 2_{i, j}\right\}$.

After subtraction of equations (4) with $K=1$ from ones with $K=2$ and elimination of terms $12_{i, j}-11_{i, j}$, the system for $g_{i, j}$ can be written as:

$$
\begin{array}{ll}
g_{i+1, j}=U_{i, j} \cdot g_{i, j} & 1 \leq i \leq N, 1 \leq j \leq N-1 \\
g_{i, j+1}=V_{i, j} \cdot g_{i, j} & 1 \leq i \leq N-1<, \quad 1<j<N, g_{1,1}=1
\end{array}
$$


where:

$$
\begin{aligned}
& U_{i j}=\left(\left|a 2_{i+1, j}-a 1_{i+1, j}\right|+w\right) /\left(\left|b 2_{i, j}-b 1_{i, j}\right|+w\right) \\
& V_{i j}=\left(\left|a 2_{i, j+1}-a 1_{i, j}+1\right|+w\right) /\left(\left|c 2_{i, j}-c 1_{i, j}\right|+w\right)
\end{aligned}
$$

and the empirical parameter $w>0$ is necessary for correct division when differences $\left(b 2_{i, j}-b 1_{i, j}\right),\left(c 2_{i, j}-c 1_{i, j}\right)$ are close to noises value $d$.

If all $g_{i, j}$ are determined then the system of equations for $f_{i, j}=\left(h_{i, j} / g_{i, j}\right)$ can be received from first $(K=1)$ or last $(K=2)$ part of (4) and written as:

$$
\begin{array}{ll}
f_{i+1, j}=f_{i, j}+q_{i, j} & 1 \leq i \leq N-1, \quad 1 \leq j \leq N \\
f_{i, j+1}=f_{i j}+p_{i j} & 1 \leq i \leq N, \quad 1 \leq j \leq N-1, f_{1,1}=0
\end{array}
$$

where:

$$
\begin{aligned}
& q_{i, j}=\left(a 1_{i+1, j} / g_{i+1, j}\right)-\left(b 1_{i, j} / g_{i, j}\right) \\
& p i_{, j}=\left(a 1_{i, j+1} / g_{i, j+1}\right)-\left(c 1_{i, j} / g_{i, j}\right)
\end{aligned}
$$

are known values.

Digital correction of any image $\left\{a_{i, j}\right\}$ for arbitrary $G$ and $H$ come to be according to expressions:

$$
I_{i, j}=G_{\cdot}\left(a_{i, j} / g_{i, j}\right)-\left(f_{i, j}+H\right) \quad 1 \leq i, j \leq N
$$

Overdetermined non-correct systems of linear equations (5) and (6) in principle can be solved by well known least squares method. According to theoretical estimates [18] this method permits to restore $\left\{I_{i, j}\right\}$ with accuracy:

$$
D \approx(\ln N)^{1 / 2} \cdot d
$$

close to detectors' noise $d$ practically for any $N$. But its numerical realization encounters some problems because of large operations' number (from $\approx N^{4}$ to $\approx N^{3}-N^{5 / 2}$ for best iterative methods) and principal limitation on computer accuracy [18 and 19].

We have developed non-iterative hierarchical method for solution systems (5) and (6) which are matched by accuracy the detectors' noise and require only $\approx 100 N^{2}$ arithmetical operations for any $N$.

Equations of system (5) can be interpreted as recurrent expressions which permit to found any $g_{i+k, j+l}$ (with $k=0,1^{i}$ and $l=0,1$ ), if $g_{i j}$ are known. Because of $2 \mathrm{D}\left\{g_{i j}\right\}$ there are many ways for such a procedure and average with using some of neighbouring ones can be done. Thus if $g 1,1=1$, then first order approximation $g_{12}(1), g_{21}(1), g_{13}(1), g_{22}(1), g_{31}(1), \ldots g_{N N}(1)$, can be obtained and new recurrent expressions for next order approximation $\left\{g_{i j}(2)\right\}$ can be written. This procedure is repeated $m \leq[\ln N]-2$ times with using every one more large-scale nets $[N / 2] .[N / 2],[N / 4] .[N / 4], \ldots\left[N / 2^{m}\right] .\left[N / 2^{m}\right]$ and more large detectors' blocks $(2.2),(4.4) \ldots\left(2^{m} .2^{m}\right)$ considered as one element, respectively. Consideration for system (6) is the same.

It is important that in conveyor regime the algorithms could be reduced up to $N^{2}$ operations.

Practical realization of the algorithms has been done by using IBM-PC/AT for 
$N<256$. Results of numerical simulation agree with theoretical estimate (8) and some of them for $N=128$ and case when $h_{i, j}=0$ are demonstrated on figure 1 .

\section{REFERENCES}

[1] LLOYD (J.M.). - Thermal imaging systems. Plenum, New-York \& London, 1975.

[2] HERRIMAN (K.H.). - Infrared imaging with linear and matrix detector arrays - a challenge to semiconductor optoelectronics. Proc. 13 Int. Symp. Techn. Comm. on Photon Meas. (Photon-Detectors). Brannschweig, 14-17 Sept. 1987, p. 207-214.

[3] Infrared detectors and arrays: critical reviews of optical science and technology. Conf. Proc., Orlando, Fla. Bellingham (Wa.), SPIE, 1988 (SPIE Proceedings, Vol. 930).

[4] VAVIVLOV (V.P.), MALDAGUE (K.) and SAPTZIN (V.M.). - Post-processing of termograms in the infrared non-destructive testing. SPIE Proceedings Infrared technology XVII, Vol. 1540, 22-26 July, 1991, San-Diego, USA.

[5] LOCKWOOD (A.H.) and PARRISH (W.J.) - Predicted performance of indium antimonies focal plane arrays. Opt. Eng., Vol. 26, No 3, 1987, p. 228-231.

[6] BALCERACK (R.), GIBSON (J.F.), GUTIERREZ (W.A.) and PILLAR (J.H.). Evolution of a new semiconductor product: mercury cadmium focal plane arrays. Opt. Eng., Vol. 26, $\mathrm{N}^{\circ} 3,1987$, p. 191-200.

[7] TOWER (J.R.). - Staring PtSi IR cameras: more diversity, more applications. Photonics Spectra, Vol. 25, No 2, 1991, p. 103-104, 106.

[8] KOSONOCKY (W.F.). - Progress in Schottky-Barrier IR images. Proc. Soc. Photo-Opt. Instrum. Eng., Vol. 750, 1987, p. 136-137.

[9] MOONEY (J.M.). - Excess low-frequency noise in PtSi on p-type Si Schottky diodes. IEEE Trans. Electron Devices, Vol. 38, No 1, 1991 p. 160-166.

[10] PERRY (D.L.) and DERENIAK (E.L.). - Proposed testing of spectral nonuniformity in PtSi area arrays [Pap.]. 1989. Proc. Soc. Photo Opt. Instrum. Eng., Vol. 1071,1989, p. 2-11.

[11] CARDONA (M.). - Modulation spectroscopy. Academic, N.Y., 1969.

[12] TIHONOV (A.N.) and ARSENIN (V.Y.). - Methods solution of non-correct problem. Moscow, Nauka, 1979 (in Russ.).

[13] TAKASHI IKUTA. - Active image processing. Appl. Opt., Vol. 24, No 18, 1985, p. 2907-2913.

[14] YARBUS (A.L.). - Role of eyes moves in vision. Nauka, Moscow, 1965 (in Russ.).

[15] MARR (D.). - Vision. Moscow, 1987 (Russ. transl.).

[16] ROSENFELD (A.); - Computer vision: a source of models for biological visual processes? IEEE Trans. Biomed. Eng., Vol. 36, No 1, 1989, p. 93-96.

[17] SAPTZIN (V.M.). - On the correction problem of non-uniformity for IR systems with arrays. Preprint Lebedev's Physical Inst. No 72, Moscow, 1989 (in Russ.). 
[18] SAMARSKIY (A.A.). - The theory of difference schemes. Nauka, Moscow, 1982 (in Russ.).

[19] YOUNG (D.M.). - A historical overview of iterative methods. Comput. Phys. Commun., Vol. 53, No 1-3, 1989, p. 1-17.

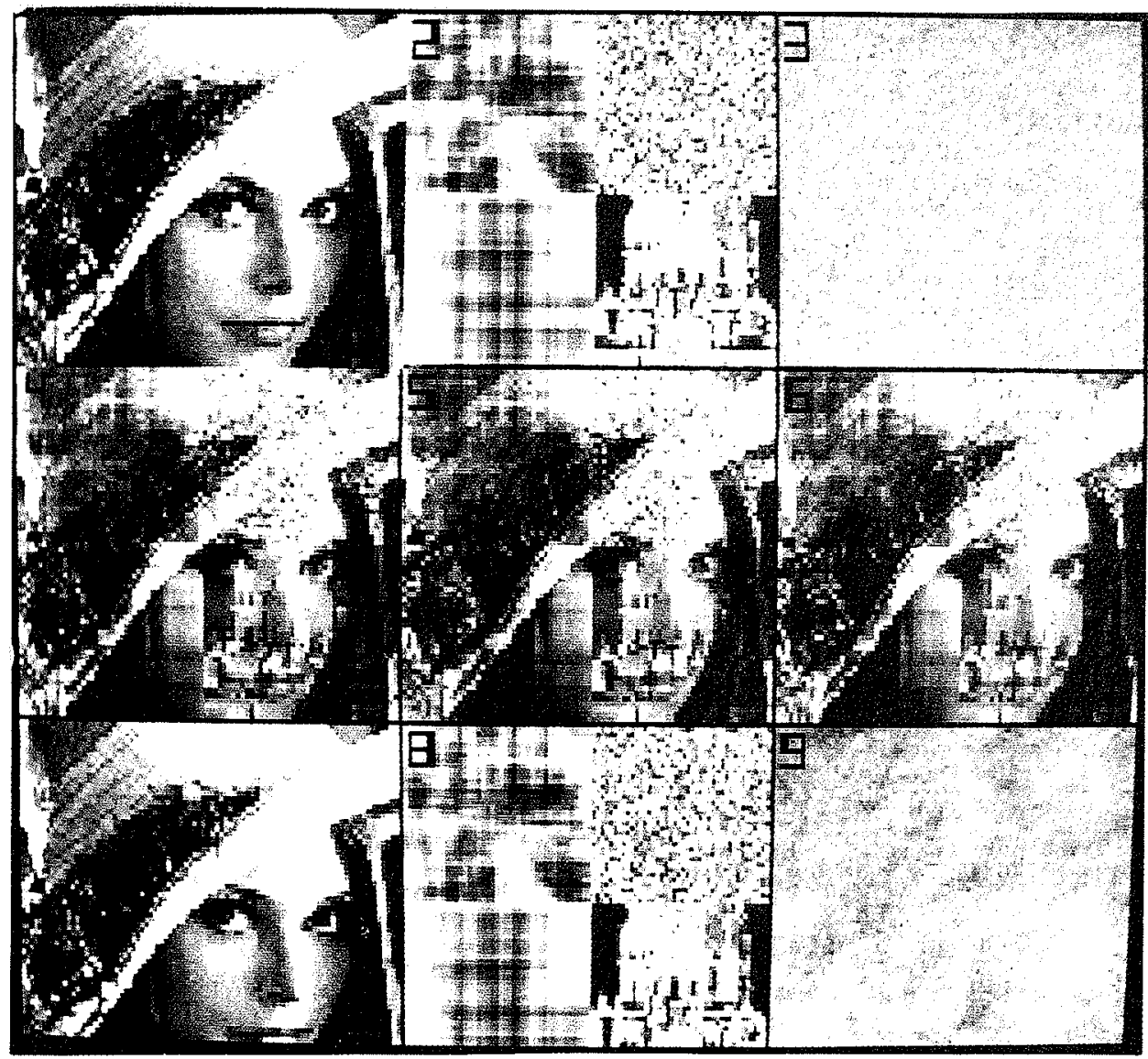

Fig.1. - Results of numerical simulation: $(1,2)$ correspond to test images $\left\{I_{i, j}\right\} \quad I_{\min }=65$, $\left.I_{\max }=216\right),\left\{g_{i, j}\right\}_{2}\left(g_{\min }=0.54, g_{\max }=1.50\right) ;(3)$ is an example of detectors' noise $\left\{d_{i, j}\right\}$
$\left(\left\langle d_{i, j}\right\rangle=0,\left\langle d_{i, j}\right\rangle=1\right)$ amplified by factor $8 ;(4-6)$ are the results of simulation for direct problem and correspond to $\left\{a_{i, j}\right\} .\left\{b_{i, j}\right\} .\left\{c_{i, j}\right\}$ with independent additive detectors' noises; $(7,8)$ are images $\left\{I_{i, j}\right\}$. $\left\{g_{i, j}^{\prime}\right\}$ restored from $(4-6)$ and $(9)$ is difference between $\left\{I_{i, j}\right\}$ and $\left\{l_{i, j}\right]$ multiplied by factor 8. 